\title{
Information System Databases for Neuropsychology Tests: Case Study in Boston Naming Test
}

\author{
Shinta Estri Wahyuningrum ${ }^{1}$, Augustina Sulastri ${ }^{2}$, Ridwan Sanjaya ${ }^{3}$ \\ Faculty of Computer Science, Soegijapranata Catholic University Indonesia ${ }^{\mathbf{1}, 3}$, Faculty of \\ Psychology, Soegijapranata Catholic University Indonesia ${ }^{2}$ \\ shinta@unika.ac,id ${ }^{1}$,ag.sulastri@unika.ac.id² ${ }^{2}$ ridwan@unika.ac.id ${ }^{3}$
}

\begin{abstract}
In the field of psychology, determining the psychological condition of a person's can be done using various types of tests. Neuropsychology test is a battery test that means every person should be taken 11 test in a moment. Each test has a different objective, as an example, The Boston Naming test is used to measure a person's ability in the language domain. The data stored for each data in the Boston Naming Test (BNT) is around 130 fields. Each test has different specific data. This makes the data grow rapidly and requires a database design that can accommodate this need.

There are many approaches can be done to store the database such a relational database and NoSQL database. When the data are stored using relational methods and amount of data are large, there can be a lack of time in both processing and tracking. This article proposes a system to store the result of the neuropsychological test using the NoSQL database approach with sample data in subtest BNT.
\end{abstract}

Keywords- Database, NoSQL, Neuropsychology, Statistical, BNT

\section{INTRODUCTION}

Nowadays there are many technologies that can be used in solving problems in various fields. One of them is web base application. Web based applications that are widely developed today can be used to process large amounts of data. When the applications are meant to be used only to handle certain amounts of data, a relational model database design is feasible to be used, but when the data to be processed is very large, complex and requires a dynamic schema change, the database that should be used is NoSQL. Applications like e- commerce, CMS and business applications are more optimal when developed with database schemes using NoSQL [1][3]. Other examples of applications using complex and a lot of data are student academic application and library application. It is complex because besides being used to store data, student and libraries applications are also accessed by many users. However, using NoSQL the database response time on a web application only takes less than one minute [2]. In Addition, NoSQL database also supports large-scale statistical analysis. In addition, NoSQL is used in EI (Em- bedded Intelligent) to process data generated from sensor readings [5].

Neuropsychology is a field of psychology that focuses on neuro-human problems. For Neuropsychological it is used name to determined the brain disorder. There are tree domain like language, attention, executive function, and memory. Each domain consists of one or more test. like a Boston Naming Test, Token Test and Verbal Fluency Test are in Language Domain. There is a benchmark used to determine a person's normal condition i.e. a normative score obtained from the mean and standard deviation of healthy people [9].

The computerized system for administering neuropsychological test data is currently being developed only in Nether- lands [10]. In Indonesia, a system is being developed to able to accommodate the needs of processing and storing data resulted from neuropsychological tests. Some constraints are encountered during the data processing. These constraints are: 1) various different tests taken by each individual. 2) All test results from each individual are documented in the database. 3) Each test has a variety of fields, even in a test 
more than 30 fields must be saved. 4) Flexibility in adding types of tests to the database. 5) Flexibility of generating normative score.

NoSQL database has a less nature schema. This allows the user to add fields to the database without changing the structure. MongoDB supports data storing using the Document database method that uses the concept of storing all data in an object databases[1]. Saved database documents can use XML, JSON, BSON document. In addition, MongoDB also supports data model flexibility and elastic scaling when they are developed into a distributed database. By using NoSQL the data storing in the database is more flexible. We can use schema-oriented, semi-structured and schemeless data [7].

\section{METHOD}

Propose system consists of two parts: to generating normative scores based on dynamic datasets and to determining patient profile.

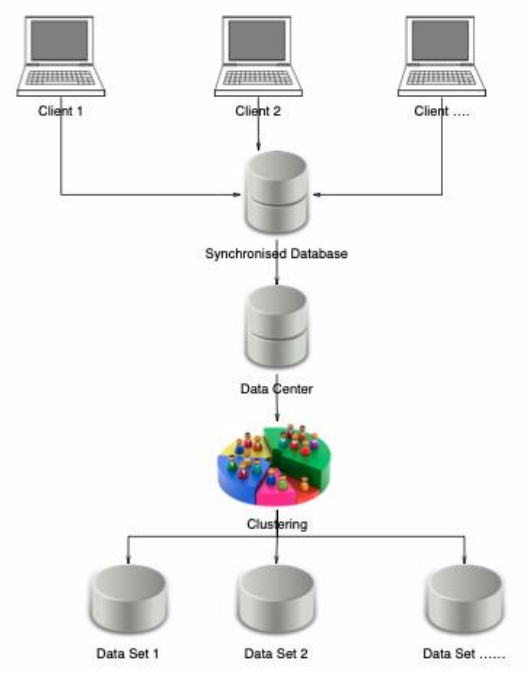

Figure 1. Center of data

\section{A. Generating normative scores based on dynamic datasets.}

Figure 1, explain how to manage the center of data. The server will receive the data from all different clients. Before the data transfer to the data center, system synchronized the raw data. The process is needed to cleaning the data, or to normalize the data. Normalize intend to make the data valid.

The data center contains data on neuropsychology results. Each participant gets a score for 11 subtests. The data of participant and score data for each sub-test will be recorded. All of data will be stored in the data center.

Data center is built to process all data into normative score information. This normative score will be standard for neuropsychologists to determine a person's performance. In addition to the data center is needed to facilitate the updating of data. Updating the data will used to get the updated of the normative score calculation. Normative data is obtained by clustering data. Normative scores are calculated for each sub-test. Normative data is calculated based on the mean and standard deviation of each sub-test. Normative scores are obtained by filtering based on several criteria from the data of participants who did the test [9]. Filtering is based on several things such as age, gender, city data collection, and education. In Figure 1 , the results of data filtering are shown in the dataset image.

\section{B. Determining patient profile}

For a neuropsychology, after obtaining a normative score, they can do a comparison of the results of a person's score with the normative score. The system built makes it easy to access data.

Patient analysis based on normative score. The output of the neuropsychology test series is to produce patients' profiles [10]. This output is obtained by comparing individual scores with a normative score that matches the patient's profile.

The neuropsychologist will input patient score results into the system show in figure 2 . Then based on patient demographic data that is detected automatically by the system, it will be compared with the results of the corresponding normative score dataset. The system will only resulted at providing data results that can be a support for experts in determining decisions. Because the ones that 
determine patient profiles are the experts, so the system will involve users, in this case neuropsychology experts, to determine the profile of the patient. If the neuropsychologist determine based on the patient's data and conclude that the patient is healthy, then the neuropsychology can submit the data to be inputted to the datacenter. The increase of data in the datacenter will be updating the normative score.

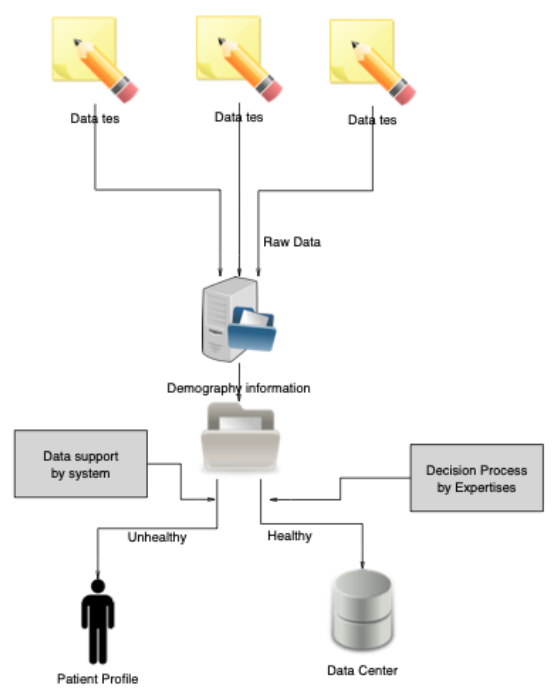

Figure 2. Access to the database

\section{Database Design}

Figure 3, shows the database design. Each test consist of a variety of different data fields, each test will be stored in a table with user id as a foreign key. Each field generated by the test should be stored in the database, to facilitate the data search process. Using the relational database design. There are 27 tables in the database design. Each table contains different field. The database is design using relational models.

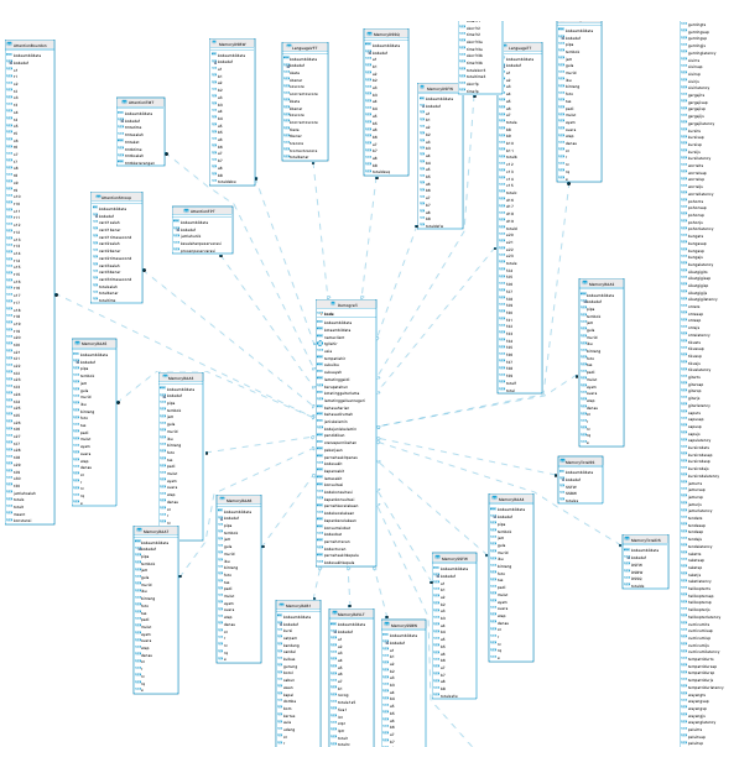

Figure 3. Table design

\section{RESULTS AND DISCUSSION}

The subtest used as sample data is the Boston Naming Test. Amount of BNT data as sample is 407 data. The database model using 407 data. Models of databases that use are MySQL model and NoSQL model.

\section{A. Database model with MySQL}

The structure table consist of two table: Demographic table and BNT score table.

Demographic tables contain detailed information from individuals including patient names, data of birth, place of residence, and history of illness. while the BNT score table contains the score data for each question in the BNT test.

The score table consists of about 300 fields for each rows. The contain od this table is data score for each question from BNT. Each question has 5 fields of score. In BNT there are 60 questions, when added with total of each fields, there will be approximately 310 fields for one rows data.

Figure 4 are the implementation of MySQL model from BNT data. There are 2 table. The LanguageBNT table has long data fields. 


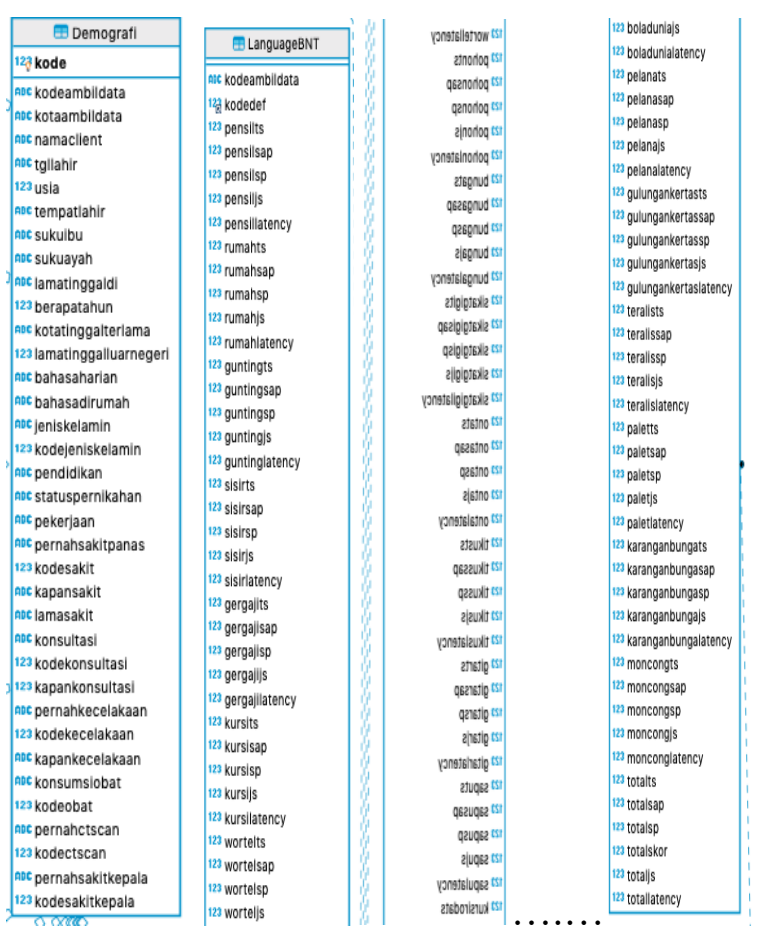

Figure 4. Database using MySQL

\section{B. Convert from MySQL to MongoDB}

The method of migrating data from MySQL to the MongoDB scheme [8] can be done in 2 ways. As can be seen in table 1, that table from MySQL will be converted to a collection in MongoDB. The first is to convert 2 tables from MySQL into 1 collection in MongoDB. In this way, BNT score data will be embedd to participant demographic data. Whereas the second way each table is convert to two tables in the colecction MongoDB. The number of table is same as in the table design with the relational method.

Table 1. Database Schema

\begin{tabular}{|l|l|}
\hline RDBMS & MongoDB \\
\hline Table & Collection \\
\hline Column & Key \\
\hline Value & Value \\
\hline Records / Rows & Document/Object \\
\hline
\end{tabular}

Conversion from two tables in MySQL to one table in NoSQL is shown in figure 5. While the databases conversion from one table in MySQL to one table in NoSQL can be seen in figure 6 .

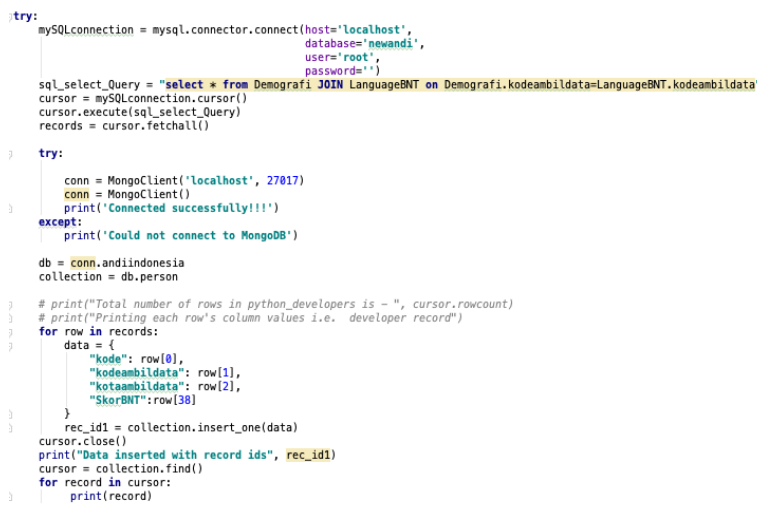

Figure 5. Convert to Embedded Collection

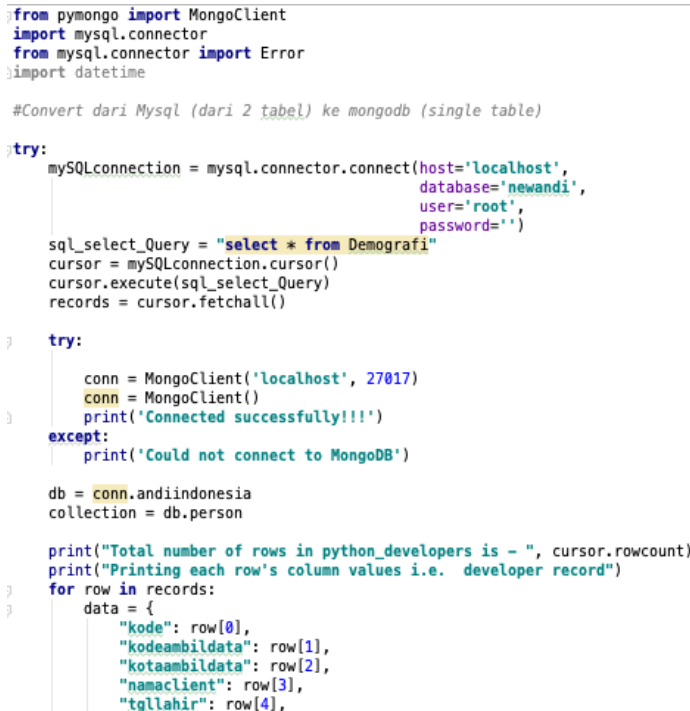

Figure 6. Convert to Single Collection

\section{Collection pada MongoDB}

After the data migration process, the data result can be seen in the figure 7 . Figure 7 is the sample of embedded collection.

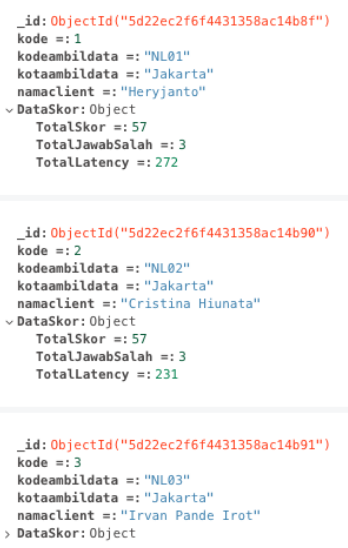

Figure 7. Embedded collection 
In the embedded collection, scores of individuals are stored in an object. In implementation using NoSQL it is possible to save different data number of fields in one collection.

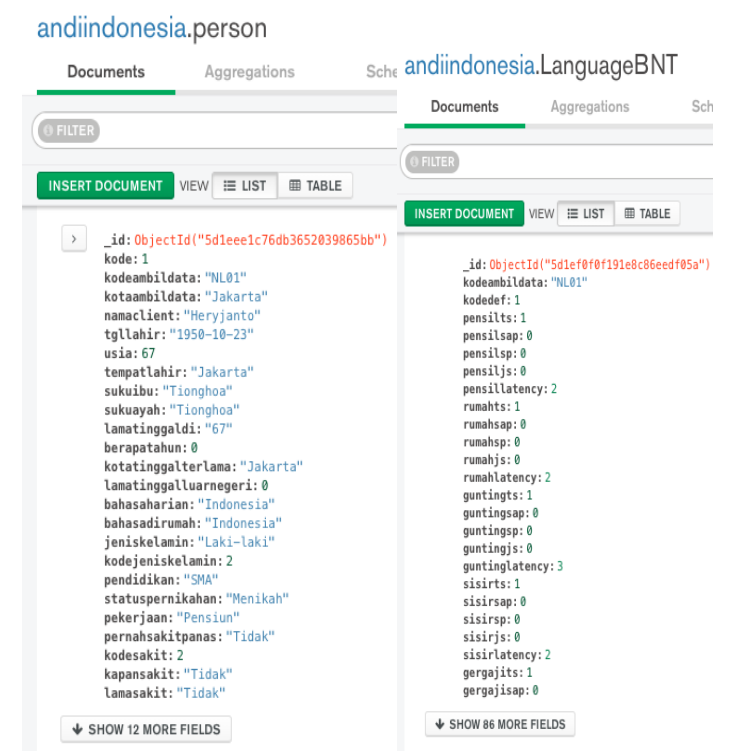

Figure 8. Single collection

Figure 8 , is a display of data stored in a single database.

The contain of table 2 is an example of a Query in the MongoDB database:

Table 2. Query in BNT NoSQL

\begin{tabular}{|c|c|}
\hline $\begin{array}{l}\text { Single } \\
\text { collect } \\
\text { ion }\end{array}$ & $\begin{array}{l}\text { andiindonesia.person.find }(\{\}) \\
\text { andiindonesia.LanguageBNT. } \\
\text { find }(\{\})\end{array}$ \\
\hline Embedded & andiindonesia.BNT.find $(\{\})$ \\
\hline Collection & $\begin{array}{l}\text { \$match }\{ \\
\text { kotaambildata: "Jakarta" } \\
\text { \$group\{ } \\
\text { _id: "\$kotaambildata", } \\
\text { total : \{ \$sum : } \\
\text { "\$dataskor.totalskor" \}\} } \\
\text { \$group\{ } \\
\text { _id: "\$kotaambildata", } \\
\text { total : \{ \$avg: } \\
\text { "\$dataskor.totalskor" \}\} } \\
\text { \$group\{ } \\
\text { id: "\$kotaambildata", } \\
\text { stddev: \{ } \\
\text { \$stdDevPop: } \\
\text { "\$dataskor.totalskor" } \\
\text { \}\} }\end{array}$ \\
\hline
\end{tabular}

Table 2, is an example of query syntax performed on the MongoDB database. The example of query use to calculate the mean and stddev values. From the query command structure, it appears that the command used to do the query is simpler.

In the implementation using a single collection, the query process that involves two tables can be done using the index based refer method. Indexes are automatically obtained when adding new data in MongoDB. The index can be seen in figure 8 in the red text. In addition to the ease of querying, adding new data to the collection is more flexible, because this will not change the structure of the collection. When new fields are added to the database, the addition of data and new fields can be done immediately when inserting new data. The addition of these fields takes effect on newly entered data, while the old data does not need to be changed and is not affected. This is rather difficult to do when using a relational database.

\section{CONCLUSION}

Using NoSQL database design makes the database flexible in storing different data for each individual. NoSQL can also be used with a large amount of data. This means the document storage system will make it faster and easier when tracking test data from each individual. In addition, if later in the development of the field of neuropsychology a new type of assessment created, it can be easily added. The advantages of designing NoSQL in BNT system are ease, speed and flexibility in determining datasets to get normative score.

\section{REFERENCES}

[1] B. Jose and S. Abraham, "Exploring the merits of nosql: A study based on mongodb", International Conference on Networks \& Advances in Computational Technologies (NetACT), Thiruvanthapuram, 2017, pp. 266-271.

[2] Z. Haishan and J. Xiaolian, "Design and Implementation of College Consumption 
Analysis System Based on NoSQL Database," 2018 International Conference on Computer Science \& Education (ICCSE), Colombo, 2018, pp. 830-834.

[3] Herrnansyah, Y. Ruldeviyani and R. F. Aji, "Enhancing query per- formance of library information systems using NoSQL DBMS: Case study on library information systems of Universitas Indonesia," 2016 International Workshop on Big Data and Information Security (IW- BIS), Jakarta, 2016, pp. 41-46.

[4] D. Ramesh, E. Khosla and S. N. Bhukya, "Inclusion of e-commerce workflow with NoSQL DBMS: MongoDB document store," 2016 IEEE International Conference on Computational Intelligence and Computing Research (ICCIC), Chennai, 2016, pp. 1-5. istep

[5] C. Yang, J. Liu, W. Hsu, H. Lu and W. C. Chu, "Implementation of Data Transform Method into NoSQL Database for Healthcare Data," 2013 International Conference on Parallel and Distributed Computing, Applications and Technologies, Taipei, 2013, pp. 198-205. ín

[6] JVD'silva, FDeMoor, BKemme, "AIDAAbstraction fo rAdvanced In-Database Analytics," 2018 Proceedings of the VLDB Endowment, 2018, pp. 1400-1413. ilép:

[7] RK Lomotey, R Deters, "Data mining from document-append NoSQL," 2014 International Journal of Services Computing (IJSC), 2014, pp. 17-29.

[8] L. Stanescu, M. Brezovan and D. D. Burdescu, "Automatic mapping of MySQL databases to NoSQL MongoDB," 2016 Federated Con- ference on Computer Science and Information Systems (FedCSIS), Gdansk, 2016, pp.

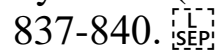

[9] Kessels R.P.C., \& Hendriks M.P.H, 'Neuropsychological Assessment. In: Howard S. Friedman," 2016 Encyclopedia of Mental Health, 2nd edition, Vol 3, pp. 197-201

[10] deVent, N. R., Rentergem, J. A., Schmand, B. A., Murre, J. M., ANDI
Consortium \& Huizenga, H. M. " Advanced Neuropsychological Diagnostics Infrastructure (ANDI): A Normative Database Created from Control Datasets, " 2017, Frontiers in

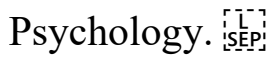

[11] Zillmer Eric A., Spiers Mary V., Culbertson William C. (2008). Principle of Neuropsychology, Second Edition. Thomson Higher Education. United States of America

[12] Zucchella Chiara, Federico Angela, Martini Alice, Tinazzi, Bartolo Michelangelo, Tamburin Stefano. (2018). Neuropsychological testing. Pract Neurol. http://pn.bmj.com/content/early/2018/02/ 22/practneurol-2017-001743\#ref-list-1

[13] Casaletto Kaitlin B and Heaton Robert K. (2017). Neuropsychological Assessment : Past and Future. Journal of the International Neuropsychological Society, 23, 778-790

[14] Alyahya, R.S.W., \& Druks, J. (2016). The adaptation of the Object and Action Naming Battery into Saudi Arabic. Aphasiology, 30 (4), 463 - 482. https://doi.org/10.1080/02687038.2015.1 070947

[15] Joao Ricardo Lourenco, Bruno Cabral, Paulo Carreiro, Marco Vieira and Jorge Bernardino. 2015. Choosing the right NoSQL database for the job : a quality attribute evaluation. Journal of Big Data. DOI 10.1186/s40537-015-0025-0

[16] Xi Zheng, Min Fu, Mohit Chugh. 2017. Big data Storage and Management in SaaS applications. Journal of Communications and Information Networks, Vol.2, No.3. DOI : 10.1007/s41650-017-0031-9 